\title{
Correspondance de La Beaumelle, éditée par Hubert Bost, Claude Lauriol et Hubert Angliviel de la Beaumelle
}

\section{Franco Piva}

\section{(2) OpenEdition Journals \\ Edizione digitale \\ URL: http://journals.openedition.org/studifrancesi/9229 \\ DOI: 10.4000/studifrancesi.9229 \\ ISSN: 2421-5856 \\ Editore \\ Rosenberg \& Sellier \\ Edizione cartacea \\ Data di pubblicazione: 1 juin 2008 \\ Paginazione: 191-192 \\ ISSN: 0039-2944 \\ Notizia bibliografica digitale \\ Franco Piva, «Correspondance de La Beaumelle, éditée par Hubert Bost, Claude Lauriol et Hubert Angliviel de la Beaumelle», Studi Francesi [Online], 154 (LII | I) | 2008, online dal 30 novembre 2015, consultato il 11 janvier 2021. URL: http://journals.openedition.org/studifrancesi/9229 ; DOI: https:// doi.org/10.4000/studifrancesi.9229}

Questo documento è stato generato automaticamente il 11 janvier 2021.

\section{cc) (†) $\odot$}

Studi Francesi è distribuita con Licenza Creative Commons Attribuzione - Non commerciale - Non opere derivate 4.0 Internazionale. 


\title{
Correspondance de La Beaumelle, éditée par Hubert Bost, Claude Lauriol et Hubert Angliviel de la Beaumelle
}

\author{
Franco Piva
}

\section{NOTIZIA}

Correspondance de La Beaumelle, éditée par Hubert BOST, Claude LAURIOL et Hubert ANGLIVIEL DE LA BEAUMELLE, avec la collaboration de Patrick ANDRIVET, Claude ANTORE et Claudette FORTUNY, t. II (1747-1749), Oxford, Voltaire Foundation, 2006, pp. XXV+466.

1 Presentando il primo volume della Correspondance de La Beaumelle (cfr. «Studi Francesi», n. 153), formulavamo l'auspicio che la realizzazione di questa importante ed imponente impresa potesse avere un ritmo abbastanza rapido, in modo da mettere presto a disposizione degli studiosi la testimonianza di questo rappresentante relativamente poco noto, ma non per questo meno significativo, per la sua posizione in qualche modo trasversale, del Settecento francese. Il nostro auspicio è stato perfettamente rispettato, in quanto, a distanza di neppure un anno, è uscito il secondo volume; il che fa bene sperare per il proseguo di questa meritevole iniziativa. Il secondo volume copre gli anni 1747-1749, vale a dire il periodo che il giovane La Beaumelle trascorse a Copenaghen come precettore del figlio maggiore del conte Carl Christian Gram, il favorito del re Federico V.

2 Per La Beaumelle (che ha appena compiuto 21 anni) è un periodo molto importante. L'esperienza danese gli apre infatti prospettive fino ad allora insospettate, anche se tutto è ancora da costruire. A questa necessità il giovane fa fronte con entusiasmo: un entusiasmo che traspare chiaramente nelle prime lettere che egli invia al padre ed al fratello. Si accinge con buona lena a svolgere il suo compito di precettore: per il giovane 
figlio del conte Gram compone una Histoire universelle e traduce in francese un'antologia di Seneca. Si adegua anche presto al ritmo, ed al tenore, che comporta la vita di corte cui la sua situazione lo obbliga a prender sempre più spesso parte. Per adeguare le sue finanze alle necessità che la vita di corte comporta, La Beaumelle tenta di farsi nominare professore dell'Accademia di Sora oppure precettore delle principesse reali, senza tuttavia riuscirci. Si rivolge allora al mondo a lui più congeniale delle Lettere: fa commercio di libri francesi, molto richiesti in un ambiente che guarda con interesse alla cultura francese, e si lancia lui stesso nel mondo della scrittura: dapprima compone L'Asiatique tolérant, la sua prima opera a stampa; poi lancia un foglio, La Spectatrice danoise, che, da un lato gli consente di esercitare il suo sguardo critico sulla realtà che lo circonda, dall'altro lo fa conoscere nel mondo delle Lettere; anche al di fuori della Danimarca, in quanto il giornale ha un certo successo.

Di questa attività le lettere comprese in questo volume parlano tuttavia poco; così come poco dicono della partecipazione di La Beaumelle alle attività della loggia massonica danese. Ai suoi parenti, ai quali sono rivolte la maggior parte delle lettere, il giovane parla soprattutto della sua vita a corte, dei suoi progetti, delle sue speranze e delle sue delusioni, assai meno delle sue esperienze nel mondo delle Lettere e della sua formazione intellettuale: come se volesse riservarsi uno spazio proprio, o se temesse di dar conto ai suoi di esperienze destinate ad emanciparlo presto dall'educazione che gli era stata impartita nel Collegio degli Enfants de Jésus o nell'Académie di Ginevra. Ma in filigrana, questa emancipazione si percepisce, così come si percepisce l'evoluzione che il giovane La Beaumelle subisce in questi anni importanti. Quello testimoniato dalle lettere raccolte in questo volume è un periodo di attesa e di passaggio, di cui lo stesso La Beaumelle fatica a rendersi conto, ma che risulterà prezioso per la sua vita futura.

Come nel primo volume, le lettere di e a La Beaumelle sono riprodotte integralmente, e nella grafia originale, mentre di quelle di o a terzi sono riportati solo i brani che lo riguardano. Le lettere sono corredate da note sobrie ma precise che consentono al lettore di conoscere più da vicino i personaggi, per lo più poco noti e appartenenti all'ambito del protestantesimo francese, coi quali La Beaumelle entra via via in contatto, oppure gli avvenimenti ai quali egli partecipa o dei quali dà testimonianza. Completano il volume una serie di documenti relativi alla vita ed all'attività di La Beaumelle sia come precettore che come scrittore, nonché l'Indice delle persone e delle opere citate. 